FUTURE PLANS AND APPROACHES 


\title{
ISO'S CAPABILITIES FOR IR BACKGROUND MEASUREMENTS
}

\author{
D. Lemke \\ Max-Planck-Institut für Astronomie \\ D-6900 Heidelberg \\ FRG
}

ABSTRACT. Although designed as a pointed infrared observatory for detailed studies of selected objects, the ISO satellite has remarkable capabilities for studying faint surface brightness sources and for helping to disentangle the overlaying background components. The 1993 launch of ISO is timely as it follows the COBE mission and offers a large guest observer program for which preparations can now be made.

\section{THE COLD OBSERVATORY}

The Infrared Space Observatory (ISO) is a fully approved and funded project of the European Space Agency (ESA). The cut-away of Figure 1 shows the major constituents. A toroidal tank containing 2300 liters of superfluid helium surrounds the $60 \mathrm{~cm}$ telescope and the baffle system. Evaporating gas cools the focal plane instruments, the telescope and the baffle to tem-

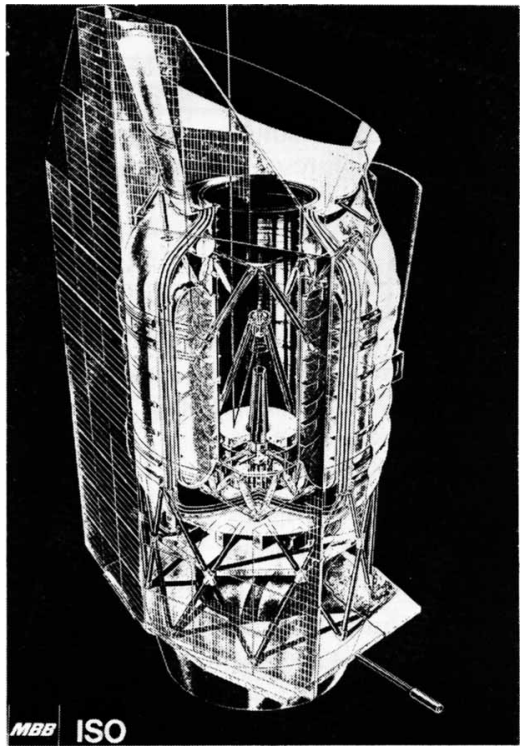

Figure 1. The ISO is cooled by 2300 liters of superfluid helium at $1.8 \mathrm{~K}$ in the main tank surrounding the $60 \mathrm{~cm}$ telescope. Sixty liters of LHe contained in the ring-shaped auxiliary tank provide cooling during the last 3 days before take-off. peratures between 3 and $7 \mathrm{~K}$. The far-IR detectors are cooled via metallic contact strips to the $1.8 \mathrm{~K}$ tank wall. With a cold telescope in space, it is possible to increase the aperture and the field of view without increasing instrumental or sky background noise.

A pyramidal mirror following the primary mirror splits the beam into four quadrants which contain the four focal plane instruments. The instruments view the sky simultaneously, but in FOVs a few arcmin apart from each other. There are two imaging photometric instruments, ISOCAM and ISOPHOT, and two spectrometers, SWS and LIVS (see Table 1). They form a coherent scientific payload capable of almost fully analyzing cosmic infrared radiation. The capabilities of the ISO instrumentation may be summarized as follows: (i) photometry, polarimetry, and imaging are possible in the wavelength range $2.5-240 \mu \mathrm{m}$; (ii) low resolution spectroscopy $(\lambda / \Delta \lambda \sim 90-1000)$ by gratings and CVFs, and high resolution 
spectroscopy $\left(-10^{4}\right)$ by Fabry-Pérots are possible in the range $2.4-180 \mu \mathrm{m}$. The satellite is capable of (i) three-axis-pointing with $\sim 2.7$ " stability, (ii) raster scanning and (iii) surveying the sky during slews.

The potential scientific targets contain all classes of objects from cometary belts, to star formation regions, to interacting galaxies. A more detailed description of ISO and the mission goals is given by Kessler (1989). The observatory will be launched in April 1993 by an Ariane $44_{p}$ Rocket and operated by the ESA Ground Observatory in Villafranca. During the 15 months of routine observation, $25 \%$ of the time will go to the Principal Investigator teams, $5 \%$ each to the Mission Scientists and the ESA observatory team, and $65 \%$ to a guest observers program.

\section{BACKGROUND STUDY CAPABILITIES}

Although ISO is designed for detailed studies of selected objects, it has several features which also make it a powerful tool for astronomical background studies, as summarized in Table 1 .

TABLE 1: ISO Instrumentation, Capabilities and Background Study Features

\begin{tabular}{|c|c|c|c|}
\hline Instrument & \multicolumn{2}{|c|}{ Wavelength Coverage $(\mu \mathrm{m})$} & PI \\
\hline $\begin{array}{l}\text { ISOCAM } \\
\text { ISOPHOT } \\
\text { SW Spectrometer } \\
\text { LW Spectrometer }\end{array}$ & \multicolumn{2}{|c|}{$\begin{array}{c}2.5-17 \\
2.5-240 \\
2.4-45 \\
45-180\end{array}$} & $\begin{array}{l}\text { Catherine Cesarsky (F) } \\
\text { Dietrich Lemke (D) } \\
\text { Thijs de Graauw (NL) } \\
\text { Peter Clegg (UK) }\end{array}$ \\
\hline \multicolumn{4}{|c|}{ ISO Capabilities } \\
\hline $\begin{array}{l}\text { Photometry } \\
\text { Polarimetry } \\
\text { Imaging }\end{array}$ & \multicolumn{2}{|l|}{$\begin{array}{l}\text { Pointing } \\
\text { Raster scanning } \\
\text { Surveying }\end{array}$} & $\begin{array}{l}\text { Low-resolution spectroscopy } \\
\text { High-resolution spectroscopy }\end{array}$ \\
\hline \multicolumn{4}{|c|}{ Relevant for Background Studies } \\
\hline \multicolumn{2}{|c|}{$\begin{array}{l}2-240 \mu \mathrm{m} \text { wavelength coverage } \\
-40 \text { selectable broad and narrow filters } \\
\text { wide-beam }\left(\sim 3^{\prime}\right) \text { photopolarimetry } \\
\text { wide-beam spectrophotometry } \\
\text { diffraction limited imaging } \\
\text { line mapping }\end{array}$} & \multicolumn{2}{|c|}{$\begin{array}{l}\text { beam blocker } \\
\text { accurate internal calibration sources } \\
\text { staring and differential (chopper) observations } \\
24 \text { hr orbit-very long integrations } \\
\text { serendipity and parallel mode } \\
\text { mission time } \geq 18 \text { month }\end{array}$} \\
\hline
\end{tabular}

The very large wavelength coverage and its division into 40 filter bands will help to disentangle the different overlaying background components by their different spectral shape. There are narrow band filters, useful if the background radiation is concentrated in certain lines such as the PAH-features (Figure 2). Other filters are placed in the "cosmological windows" at the 4 and $50 \mu \mathrm{m}$ minima of the nearest foreground emission of the interplanetary and interstellar dust emission. Wide beam observations are possible in single beams up to $110 \mu \mathrm{m}$, and by co-adding the pixels of the 6 cameras covering the region 2.5 to $240 \mu \mathrm{m}$ (Figure 3). The largest FOV achievable is $3^{\prime}$ and is determined by the maximum size of the unvignetted FOV. Larger fields can be covered by mapping techniques. Both spectrometers could map certain regions in strong emission lines; they have to be operated at fixed apertures of 99" (LWS) and $14 \times 20$ or $20 \times 30 \operatorname{arcsec}^{2}$, depending on wavelength (SWS). 


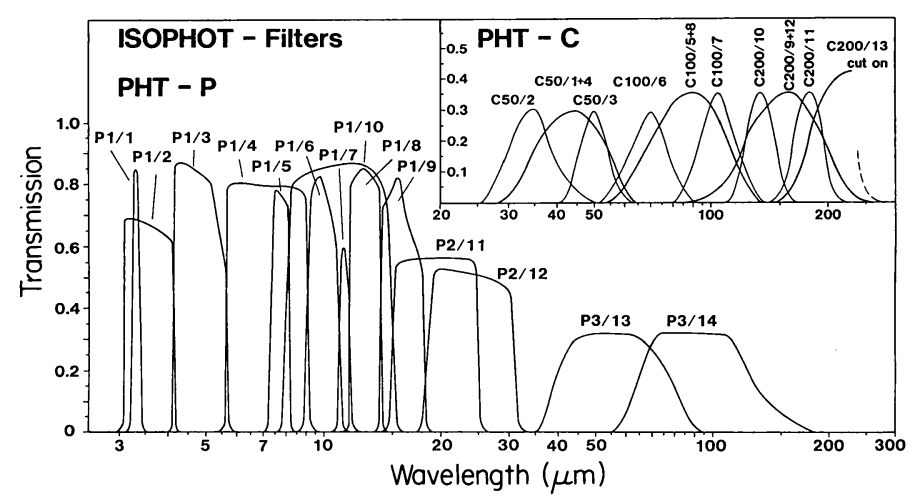

Figure 2. Broad and narrow band filters of ISOPHOT. ISOCAM has a similar number of filters and a CVF. All filters can be combined with polarizers.

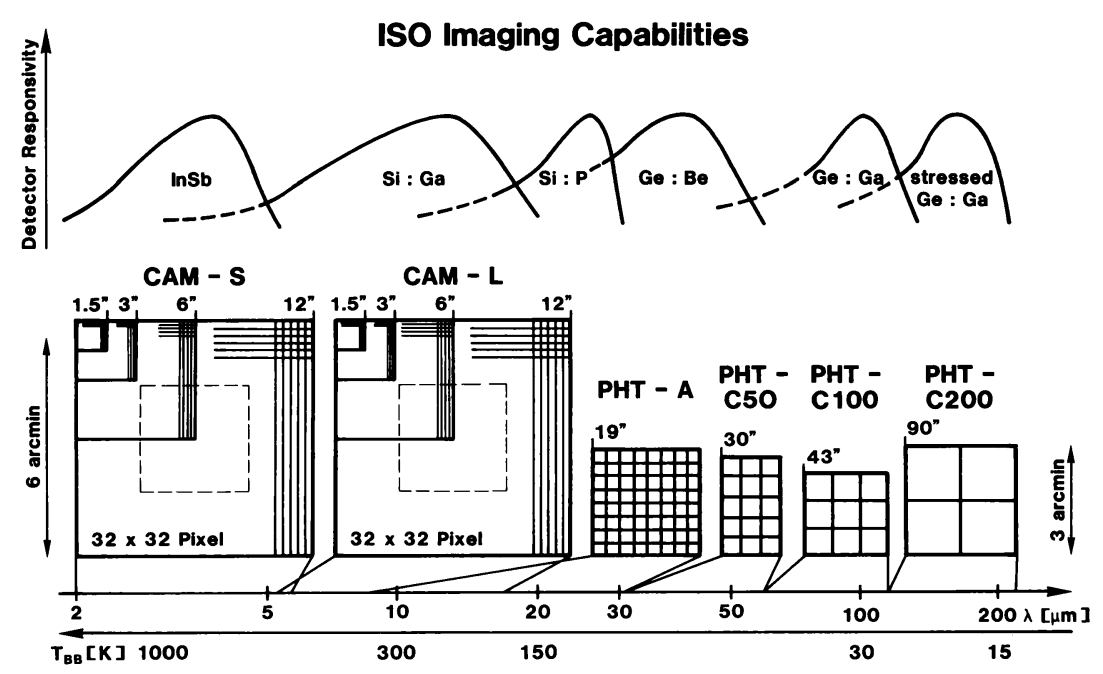

Figure 3. ISO's imaging capabilities are contained in ISOCAM $(\lambda<17 \mu \mathrm{m})$ and ISOPHOT $(10-240$ $\mu \mathrm{m})$. ISOCAM can select the plate scale by a lens wheel. The unvignetted FOV is always limited to $3^{\prime}$ by a field mirror inside the camera (dotted square).

Of high importance in cosmological background studies is the flux calibration. All instruments are equipped with internal calibration sources, some share identical filters in order to allow intercomparison. The temperature stability of ISOPHOT's calibration source is $\Delta T \sim 0.01 \mathrm{~K}$. Most instruments have a beam blocker in order to be able to frequently check the zero points. ISOPHOT is equipped with a focal plane chopper allowing accurate differential measurements.

On-target integration times of many hours are possible on the highly eccentric $24 \mathrm{hr}$ orbit, and these are needed for the faintest background sources. During the long mission time of $\geq 18$ months most targets will be accessible several times and therefore enable the study of, or correction for, variability as in the case of the seasonal variations of the zodiacal emission. 


\section{INSTRUMENT SENSITIVITY}

The minimal detectable flux for both pointlike and extended objects is plotted in Figure 4. This flux refers to a $\mathrm{S} / \mathrm{N}=10$ and $100 \mathrm{~s}$ integration time and is given for ISOPHOT. In the

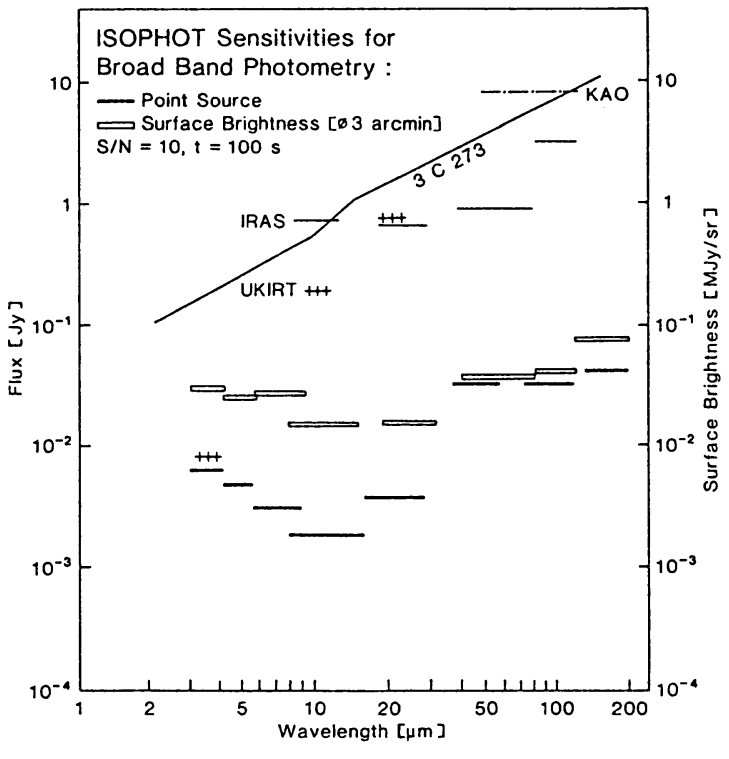

Figure 4. ISOPHOT's minimal detectable flux. overlapping region $2.5-17 \mu \mathrm{m}$, ISOCAM achieves a similar performance. While for pointlike sources a comparison can be made to the IRAS survey limits in the same figure, the surface brightness values can be compared with the natural backgrounds in Figure 5. Indicated is the brightness of the cirrus clouds as derived from IRAS by Boulanger and Perault 1988. A filter spectrophotometry of these faint clouds whose spectrum is expected to be similar to that of the bright reflection nebula NGC2023 (Figure 6) can be made in $-500 \mathrm{~s}$ (Lemke et al. 1989). While all sensitivity numbers of Figure 4 and 5 refer to the widest beams, the aperture size effect is given as an example of the $11 \mu \mathrm{m}$ broadband filter in Figure 7.

Another example on the edge of ISO's capabilities is indicated in

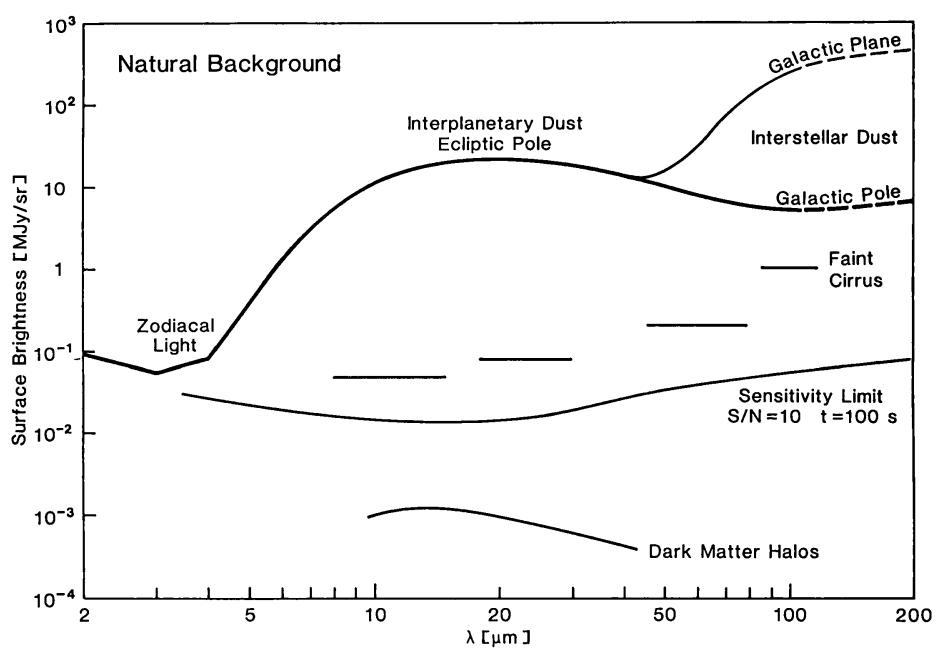

Figure 5. Natural background flux and ISOPHOT sensitivity. 


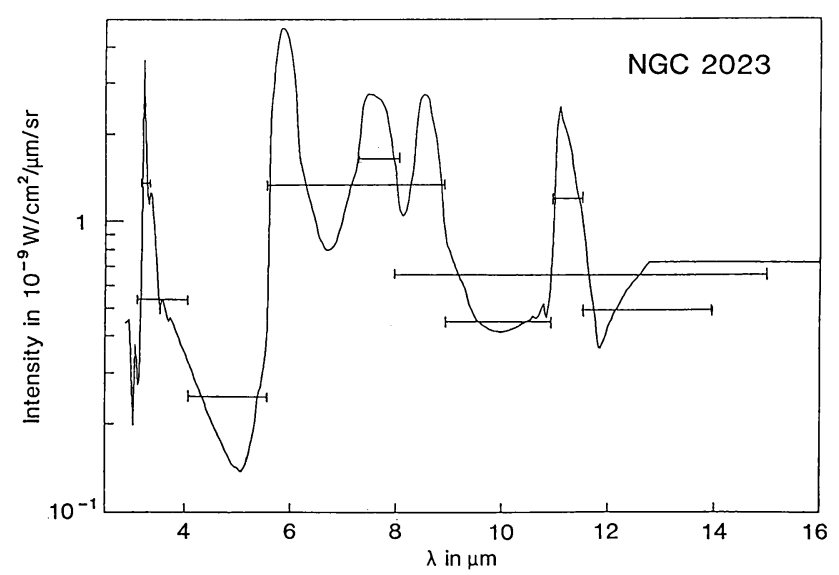

Figure 6. Spectrum of the bright reflection nebula NGC2023 measured by an airborne telescope by K. Sellgren. The features are thought to be caused by polycyclic aromatic hydrocarbons (PAHs). ISOPHOT's filters are placed so as to study these faint extended sources.

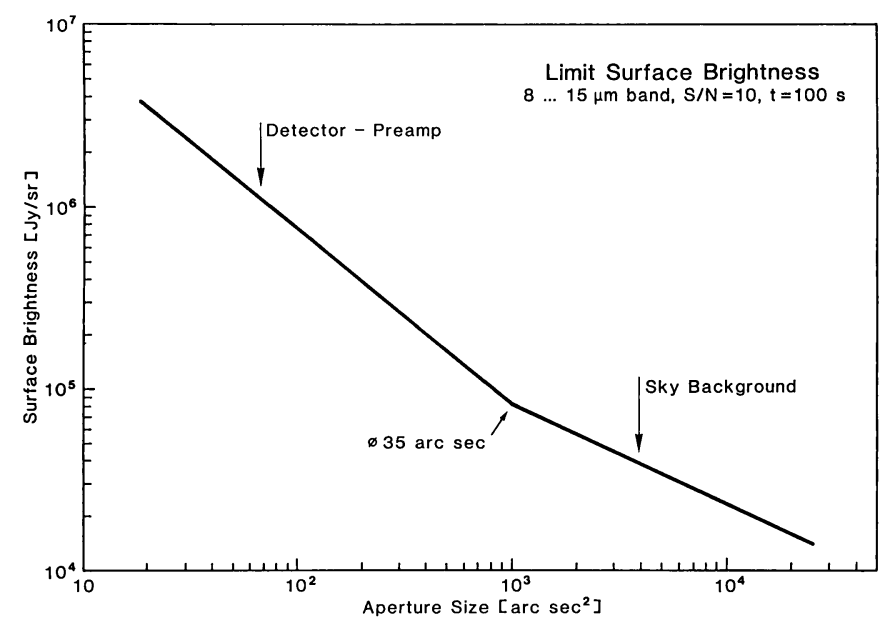

Figure 7. Surface brightness sensitivity of ISOPHOT in the $11 \mu \mathrm{m}$ broad band filter versus the selectable aperture size. The change in the curve's steepness is caused by the change in the limitation due to detector/preamp noise and the zodiacal light photon background fluctuations.

Figure 5: the dark matter halos. This "galactic background radiation of other galaxies" is supposed to be caused by the combined light of brown dwarfs forming massive halos. Observations with ISO's wide beam on edge-on-galaxies could reveal the halo radiation if they exist and current models are correct (for a summary see Jura 1988). The cirrus in the galaxy under investigation and in our galaxy is competing with the integrated brown dwarf radiation. Modelling for the stellar contribution, the zodiacal light, etc., as well as broad and narrowband photometry and many hours of observing time will be needed in order to confront the problem of dark matter halos. Despite all these difficulties one has to try this observation, which is for the first time within reach of an observatory. 


\section{SERENDIPITY AND PARALLEL MODE}

The otherwise wasted dead time during slews of the satellite from target to target will be used to perform the first sensitive $200 \mu \mathrm{m}$ sky survey by ISOPHOT's $200 \mu \mathrm{m}$ camera. With a slew

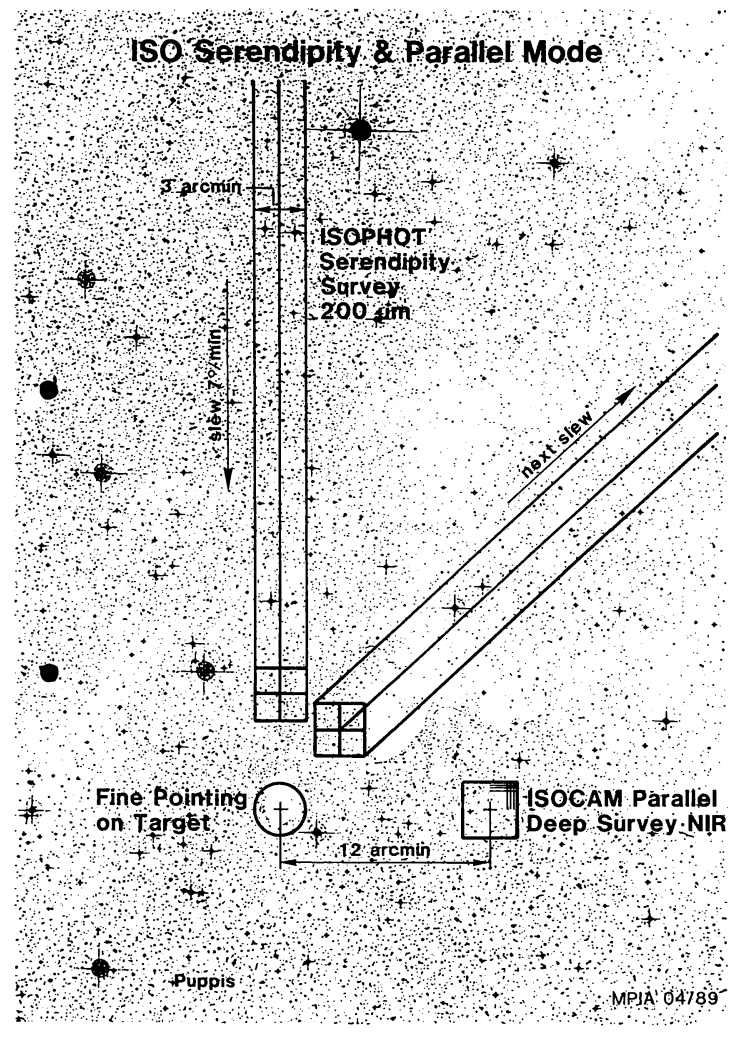

speed of up to $7^{\circ} \mathrm{min}^{-1}$, the limiting magnitude in these serendipitous strip maps is expected to be $\sim 1 \mathrm{Jy}$. The results will complement the IRAS sky survey $(\lambda<110 \mu \mathrm{m})$ and explore the large scale FIR background radiation. The sky coverage and redundancy can only be calculated after a model observing plan is established. It is estimated that $10-40 \%$ of the sky can be explored.

During long exposures of any of the other instruments, ISOCAM can make deep observations of the sky close to the target in the near-IR. Both additional modes are illustrated in Figure 8.

Figure 8. ISOPHOT's $200 \mu \mathrm{m}$ camera will survey the sky during the satellite's slews, while ISOCAM takes deep exposures in the near- $\mathbb{R}$ during long on-target integrations of other experiments.

Acknowledgements-I thank Dr. M. Kessler, ISO Project Scientist ESA, for a critical reading of the manuscript, and Dr. L. J. Puget, ISO Mission Scientist, for interesting discussions on the interstellar dust component. E. Ackermann, M. Burgdorf and Ch. Hajduk of MPI Astronomie contributed to the sensitivity calculations performed by an ISOPHOT instrument simulation S/W.

\section{REFERENCES}

Boulanger, F., and Perault, M. 1988, Astrophys. J., 330, 964.

Jura, M. 1988, Astro. Lett. and Comm., 27, 113.

Kessler, M. 1989, Proc. SPIE, 1130, in press.

Lemke, D., Krätschmer, W., Tuffs, R., Kreysa, E., and Léger, A. 1989, Proc. of 22. ESLABSymposium, Salamanca 1988, ed. M. Kessler, in press. 